Check for updates

Cite this: RSC Adv., 2017, 7, 38220

Received 16th May 2017

Accepted 10th July 2017

DOI: $10.1039 / \mathrm{c} 7 \mathrm{ra05506d}$

rsc.li/rsc-advances

\section{Preparation of carbon aerogels from TEMPO- oxidized cellulose nanofibers for organic solvents absorption $\dagger$}

\begin{abstract}
Meng Wang, (DD a Changyou Shao, ${ }^{a}$ Sukun Zhou, ${ }^{a}$ Jun Yang ${ }^{\star a b}$ and Feng $\mathrm{Xu}^{\star a}$
In this study, ultralight and hydrophobic carbon aerogels were prepared from 2,2,6,6-tetramethyl-1piperidinyloxy (TEMPO)-oxidized cellulose nanofibers (TOCN) for the removal of organic solvents. The morphological structure, density and chemical composition of TOCN aerogels frozen at $-56{ }^{\circ} \mathrm{C}$ and $-196{ }^{\circ} \mathrm{C}$, followed by pyrolysis, were examined. The obtained carbon-based aerogels possessed an ultralight density of $8.8 \mathrm{mg} \mathrm{cm} \mathrm{m}^{-3}$ and excellent fire-resistance. The high porosity (up to $99.5 \%$ ) and hydrophobicity (contact angle of $139.6^{\circ}$ ) of the obtained carbon aerogels allowed them to adsorb various organic solvents with a high absorption capacity (up to $110-260 \mathrm{~g} \mathrm{~g}^{-1}$ ). After five absorptioncombustion and absorption-distillation cycles, the absorption capacity of the TOCN carbon aerogels exhibited excellent recyclability with up to $64-99 \%$ of the initial absorption capability, demonstrating potential applications in the oil-spill-cleanup field.
\end{abstract}

\section{Introduction}

Owing to increased attention focused on marine pollution resulting from organic pollutants or oil spills, much effort has been made to produce efficient and economic absorbents, which can adsorb and separate organic pollutants from water. ${ }^{1,2}$ Oil recovery using absorbent material is superior to several other industrial methods for oil recovery, such as chemical methods (i.e., dispersion, in situ burning, and the use of solidifiers), physical collection, and biodegradation, because of the lower costs, higher efficiency and excellent recyclability of most absorbents. ${ }^{3}$ Recently, people have paid much attention to the development of porous materials as absorbents because of their high oil-water separation properties. Various natural materials such as expanded perlite ${ }^{4}$ and zeolites ${ }^{5}$ and organic materials such as wool fiber, ${ }^{6}$ activated carbon, ${ }^{7}$ and sawdust have been reported as porous adsorbents for cleaning oil spills. ${ }^{8}$

Carbon-based aerogels, composed of interconnected threedimensional (3D) networks, have attracted wide attention because of their unique physical properties, such as ultra-low density, high electrical conductivity, continuous porosity, and

${ }^{a}$ Beijing Key Laboratory of Lignocellulosic Chemistry, Beijing Forestry University, Beijing 100083, China. E-mail: xfx315@bjfu.edu.cn; yangjun11@bjfu.edu.cn; Fax: +86-10-62336903; Tel: +86-10-62337993; +86-10-62337223; +86-10-62336387

${ }^{5}$ State Key Laboratory of Pulp and Paper Engineering, South China University of Technology, Guangzhou 510640, China

$\dagger$ Electronic supplementary information (ESI) available: Video S1: the video shows that a TOCN carbon aerogel was immersed into the $n$-hexane water mixture $(1: 20$ $\mathrm{v} / \mathrm{v}$, colored with Sudan III). Video S2: as shown in the video, the chloroform (stained with Sudan III) lying at the bottom of the water can be absorbed by the TOCN carbon aerogels. See DOI: 10.1039/c7ra05506d high specific surface area., ${ }^{9,10}$ These properties offer numerous benefits for carbon-based aerogels as functional materials. ${ }^{11}$ Particularly, their 3D cross-linked networks with open pores allow access and fast diffusion of molecules into the material, providing the aerogels with excellent performance as super absorbents, ${ }^{12}$ gas sensors,${ }^{13}$ electrode materials for batteries and super-capacitors, ${ }^{14}$ catalyst supports, ${ }^{15}$ and chemical and biological sensors. ${ }^{16}$

Traditionally, to fabricate carbon aerogels, resorcinol-formaldehyde organic aerogels are pyrolyzed in an inert atmosphere to form a highly cross-linked carbon structure, which possess a relatively high density $\left(100-800 \mathrm{mg} \mathrm{cm}{ }^{-3}\right) \cdot{ }^{17}$ Nowadays, carbon nanotube (CNT) sponges ${ }^{18}$ and their derivatives, have been prepared through chemical vapor deposition (CVD) with high sorption capacity. However, the harmful and expensive precursors, complicated process, and complex equipment involved in CNT fabrication hamper their large-scale production for industrial applications, which pushes us to explore a facile, economic and environmentally friendly strategy for mass production of new carbon-based aerogels.

Nanocellulose is one of the nanoscale building blocks prepared from disintegration of cellulose, and it shows particular promise for use in aerogel materials because of its sustainability, high strength, low density, liquid-crystalline properties, biodegradability, and general biocompatibility. ${ }^{19,20}$ Cellulose nanofibers can be disintegrated from the hierarchical structure of the macroscopic wood fibers via several methods, such as mechanical treatments, chemical treatments and chemo-mechanical methods. Since 2,2,6,6-tetramethyl-1piperidinyloxy (TEMPO)-oxidized cellulose nanofibers (TOCNs) are made from native wood celluloses by TEMPO-mediated 
oxidation and successive mild disintegration, they always exhibit long aspect ratios (60-100), making them very flexible and easily entangled. When interwoven, TOCN can form highly porous and mechanically strong materials such as nanocellulose papers, films and aerogels. ${ }^{21,22}$

Although the aerogels prepared from cellulose have been widely documented, the transformation of cellulose aerogels into their carbonaceous forms has been less reported., ${ }^{\mathbf{9} 18}$ Recently, carbon aerogels were produced from winter melon for recyclable oil absorption. However, the attained carbon aerogels showed low performance in sorption of oils and organic solvents (15-60 times). ${ }^{23}$ To date, there is a trend to produce carbon-based materials from biomass materials, as it is very cheap, easy to obtain, sustainable, and environmentally friendly, which further inspires us to explore TOCN carbon aerogels. ${ }^{23-25}$

In this study, 3D carbon aerogels were made by freeze-drying a suspension and post-pyrolyzing the aerogel using TOCN as the raw material. Considering that the freezing temperature would significantly affect the ice formation rate, we compared the effect of freezing temperature $\left(-56\right.$ and $\left.-196{ }^{\circ} \mathrm{C}\right)$ on the morphology and physical properties of TOCN carbon aerogels. The ultralight, highly porous and hydrophobic carbon aerogels can selectively separate and remove oil and toxic organic solvents from water. It is expected that with a combination of low-cost biomass as a raw material, green preparation processes and outstanding recyclability, the TOCN carbon aerogels have promising applications in environmental and ocean protection.

\section{Experimental}

\section{Materials}

A commercial softwood kraft pulp was provided by Northwood Pulp and Timber Limited (Canada). The pulp was thoroughly washed with deionized water and used in its never-dried form. The 2,2,6,6-tetramethylpiperidin-1-oxyl (TEMPO) was purchased from Sigma-Aldrich. Sodium bromide, sodium hydroxide and sodium hypochlorite solution were received from Lanyi Medicine (Beijing). All chemicals were analytical grade and used without further purification. Ultrapure water was produced by a Heal Force purification system and used to prepare all aqueous solutions.

\section{Preparation of the TOCN aerogels}

TOCN suspensions were prepared from softwood kraft pulp according to the previously published method. ${ }^{26}$ The cellulose fibers $(1 \mathrm{~g})$ were suspended in water $(100 \mathrm{~mL})$ containing TEMPO (0.016 g, $0.1 \mathrm{mmol})$ and sodium bromide $(0.1 \mathrm{~g}, 1$ $\mathrm{mmol}$ ). The $12 \% \mathrm{NaClO}$ solution was adjusted to $\mathrm{pH} 10$ by the addition of $0.1 \mathrm{M} \mathrm{HCl}$. The TEMPO-mediated oxidation was started by adding the desired amount of the NaClO solution (5.0 mmol NaClO per gram of cellulose) and continued at room temperature while stirring at $500 \mathrm{rpm}$. The $\mathrm{pH}$ was maintained at 10 by adding $0.5 \mathrm{M} \mathrm{NaOH}$ using a $\mathrm{pH}$ meter until no $\mathrm{NaOH}$ consumption was observed. The TEMPO-oxidized cellulose was thoroughly washed with water via centrifugation. Then, the
TEMPO-oxidized cellulose suspension was fibrillated by a highpressure homogenizer (APV-2000, $100 \mathrm{MPa}$ ) for $20 \mathrm{~min}$. Thereafter, the TOCN suspensions were adjusted in concentration to $0.5,1,1.5,2 \mathrm{wt} \%$ and stored at $4{ }^{\circ} \mathrm{C}$ before further treatment or analysis. After being transferred into a plastic beaker, the cellulose suspensions were frozen under two different temperatures: one was subjected to $-196{ }^{\circ} \mathrm{C}$ in liquid nitrogen for $20 \mathrm{~min}$ and the other to $-56{ }^{\circ} \mathrm{C}$ in an ultralow temperature refrigerator for $20 \mathrm{~min}$. Then, the frozen samples were dried using a freeze-dryer (Scientz-10N, China) and the sponge-like TOCN aerogels were obtained. During freeze-drying, the cold trap temperature was below $-56{ }^{\circ} \mathrm{C}$ and the vacuum was below 0.08 mbar. The obtained TOCN aerogels frozen at $-196{ }^{\circ} \mathrm{C}$ and $-56{ }^{\circ} \mathrm{C}$ were named $\mathrm{T} 1$ and $\mathrm{T} 2$, respectively.

\section{Preparation of the TOCN carbon aerogels}

The obtained TOCN aerogels $(\sim 0.2 \mathrm{~g})$ were transferred into a tubular furnace for pyrolysis under nitrogen flow $(30 \mathrm{~mL}$ $\min ^{-1}$ ). The TOCN aerogels were heated to $500{ }^{\circ} \mathrm{C}$ at a heating rate of $2{ }^{\circ} \mathrm{C} \min ^{-1}$, kept at this temperature for $1 \mathrm{~h}$, heated to $1000{ }^{\circ} \mathrm{C}$ at a heating rate of $5{ }^{\circ} \mathrm{C} \mathrm{min}^{-1}$ and held at this temperature for $2 \mathrm{~h}$ to allow complete pyrolysis. Then, the sample was cooled to room temperature naturally, after which the samples of $\mathrm{T} 1$ and $\mathrm{T} 2$ post-pyrolysis were designated $\mathrm{C} 1$ and $\mathrm{C} 2$, respectively.

\section{Characterization}

TOCN suspensions were dispersed in ethanol and ultrasonicated for $10 \mathrm{~min}$. The homogeneous suspensions were collected using carbon film-covered copper grids and stained with phosphotungstic acid ( $2 \mathrm{wt} \%$ ) for $10 \mathrm{~s}$. These were then observed using transmission electron microscopy (TEM JEM-1010) at an accelerator voltage of $80 \mathrm{kV}$. The surface morphology of the TOCN aerogels and the carbon aerogels were observed by scanning electron microscopy (SEM, S-3400 N II). The fractured specimens were attached to the holders with conductive double-sided carbon tape and sputter coated with gold to avoid charging during the observation. The Fourier transform infrared spectroscopy (FTIR) spectra were measured using an infrared spectrophotometer (Nicolet iN10-MX, ThermoScientific). XRD patterns of the aerogels and carbon aerogels were recorded using a D8-Advance X-ray diffraction analyzer. X-ray photoelectron spectroscopy (XPS) measurements were performed on a Thermo ESCALAB 250 using a monochromic Al X-ray source. Specific surface area was calculated using the Brunauer-Emmett-Teller (BET) equation, and the pore size distribution plot was determined by the Barrett-JoynerHalenda (BJH) theory (SSA-700, Beijing, China). The wetting properties of different samples were evaluated through contact angle tests, which were performed by the CAST2.0 contact angle analysis system at room temperature (Solon Information Technology Co, Ltd, Shanghai, China).

\section{Density}

The TOCN aerogels and carbon aerogels were cut to an approximately $1 \mathrm{~cm}^{-3}$ volume. The dimensions and mass were measured using a digital caliper and balance to $0.01 \mathrm{~mm}$ and 
$0.1 \mathrm{mg}$ resolution, respectively, in order to calculate the density of the aerogels.

\section{Porosity}

The porosity of the aerogels $(P)$ was calculated by the density of the aerogels $\left(\rho_{\text {gel }}\right)$ and the density of the solid $\left(\rho_{\text {solid }}\right)$ as follows:

$$
P(\%)=100 \times\left(1-\frac{\rho_{\text {gel }}}{\rho_{\text {solid }}}\right)
$$

\section{Absorption capacity}

The absorption capacity of TOCN carbon aerogels was measured by immersing a piece of TOCN carbon aerogel $(\sim 1$ $\mathrm{cm}^{3}$ ) in $30 \mathrm{~mL}$ organic solvents for $10 \mathrm{~min}$ and allowing it to saturate. The saturated carbon aerogel was then taken out for mass measurement. The organic solvents absorption capacity ( $\mathrm{g}$ $\mathrm{g}^{-1}$ ) of TOCN carbon aerogels was calculated as follows:

$$
Q_{\mathrm{s}}=\frac{\left(m_{\mathrm{st}}-m_{\mathrm{o}}\right)}{m_{\mathrm{o}}}
$$

where $Q_{\mathrm{s}}$ is the absorption capacity and $m_{\mathrm{st}}$ and $m_{0}$ are the weights of fully saturated and dry carbon aerogels, respectively.

\section{Adsorption kinetics}

In order to further research the oil absorption kinetics of TOCN carbon aerogels, carbon aerogels adsorbed with gasoline and diesel oil were investigated. The carbon aerogels were taken out from the oil and weighed at several time intervals.

\section{Results and discussion}

\section{TOCN and its aerogel morphology}

To produce cellulose fibers with high aspect ratios, softwood pulp fibers were used as the raw material in this study. Cellulose nanofibrils with an average width of 2-5 $\mathrm{nm}$ and lengths of up to several micrometers were prepared via coupled TEMPO mediated oxidation and homogenization (Fig. 1b and inset). After homogenization, the oxidized cellulose slurries were mostly converted to highly viscous dispersions and were stable for several months under ambient conditions (Fig. 1a). Indeed, the mechanical disintegration makes the cellulose fibers entangle with each other to form a denser network structure (Fig. 1b).

The TOCN suspensions were adjusted to concentrations of $0.5,1,1.5$ and $2 \mathrm{wt} \%$; the density of the subsequent aerogels and carbon aerogels from these suspensions are shown in Table 1. The $\mathrm{C} 1$ and $\mathrm{C} 2$ prepared from $0.5 \mathrm{wt} \%$ concentration of TOCN had the lowest density and highest oil absorption but were fragile after absorption of oil (Table 2). The C1 and C2 prepared from 1.5 and $2 \mathrm{wt} \%$ concentration of TOCN possessed high density and low adsorption capacity (Table 2). As the porosity of TOCN carbon aerogel reduces, there is less space in the aerogel network for oil occupation, and thus the oil adsorption capacity
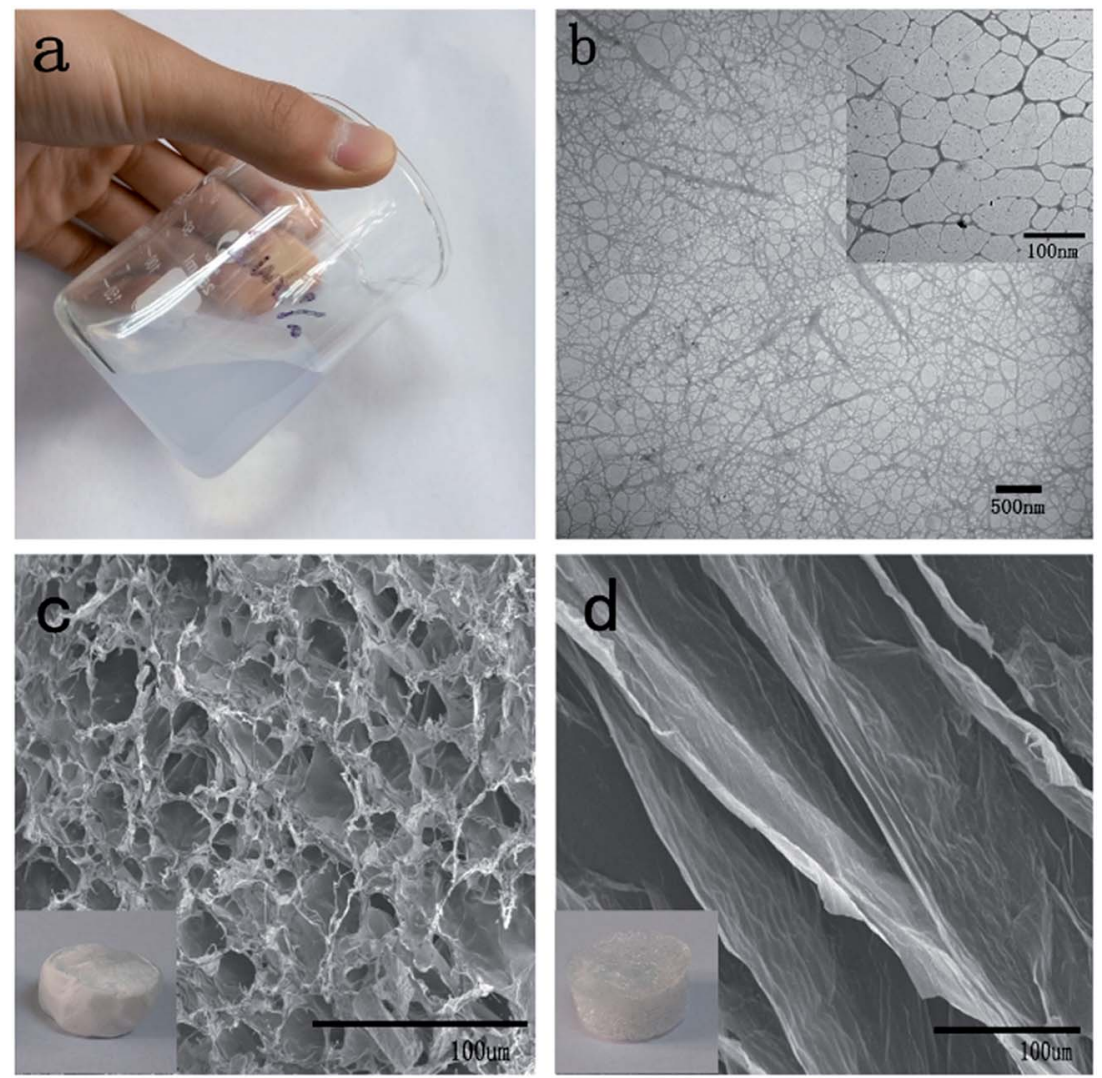

Fig. 1 (a) The highly viscous dispersions of 1 wt $\%$ cellulose gel. (b) TEM image of the TOCN. (c) and (d) TOCN suspensions frozen at $-196{ }^{\circ} \mathrm{C}$ in liquid nitrogen and $-56^{\circ} \mathrm{C}$ in an ultralow temperature refrigerator followed by freeze drying to form aerogels. 
Table 1 Density of TOCN carbon aerogels from different TOCN concentrations

TOCN concentration

\begin{tabular}{lllll} 
(wt\%) & 0.5 & 1 & 1.5 & 2 \\
\hline
\end{tabular}

$\mathrm{T} 1\left(\mathrm{mg} \mathrm{cm}^{-3}\right)$

$\mathrm{T} 2\left(\mathrm{mg} \mathrm{cm}^{-3}\right)$

C1 $\left(\mathrm{mg} \mathrm{cm}^{-3}\right)$

$\mathrm{C} 2\left(\mathrm{mg} \mathrm{cm}^{-3}\right)$

$\begin{array}{rr}5 \pm 0.1 & 10 \pm 0.2 \\ 5 \pm 0.1 & 10 \pm 0.2 \\ 4.4 \pm 0.1 & 8.8 \pm 0.2 \\ 2.9 \pm 0.1 & 5.8 \pm 0.1\end{array}$

$15 \pm 0.2$

$15 \pm 0.2$

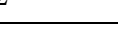

lowers. Therefore, we finally chose a concentration of $1 \%$ as our final experimental concentration.

The TOCN suspension $(1 \mathrm{wt} \%)$ was frozen at $-196{ }^{\circ} \mathrm{C}$ and $-56{ }^{\circ} \mathrm{C}$, respectively, and freeze-dried to form aerogel. The results show that both $\mathrm{T} 1$ and $\mathrm{T} 2$ aerogels had similarly low densities (about $10 \mathrm{mg} \mathrm{cm}^{-3}$ ). However, the surface of $\mathrm{T} 1$ was more smooth than that of T2 (inset, Fig. 1c and d). As shown in SEM images, T1 appeared to contain numerous small pores (Fig. 1c), but T2 formed large sheet-like structures (Fig. 1d). This morphological distinction between $\mathrm{T} 1$ and $\mathrm{T} 2$ may be predominately attributed to the different freezing rates. Before freezing, the nanofibers were uniformly dispersed in water. During the freezing process, owing to the squeezing effect, the nanofibers at the boundary of the ice crystals were concentrated and aligned along the growth direction of the ice crystals. ${ }^{27}$ According to the reverse relationship between the ice nuclei sizes and the cooling rate, as the suspension was immersed in liquid nitrogen, the water in the surface of the suspension was frozen immediately. Due to the extremely low temperature, the ice crystallized quickly and the crystal size was small, leading to small pores in T1 (Fig. 1c). In contrast, for the specimens frozen at $-56{ }^{\circ} \mathrm{C}$, the ice crystals formed much more slowly, leading to the sheet-like structures seen in $\mathrm{T} 2$. Therefore, the larger ice crystal size in samples frozen at $-56{ }^{\circ} \mathrm{C}$ resulted in the large sheet-like structure of $\mathrm{T} 2$.

\section{Carbon aerogel morphology and properties}

After pyrolysis, as shown in Fig. $2 \mathrm{a}$ and $\mathrm{b}$, the volume of T1 and T2 shrank by about $75 \%$ and $62 \%$, respectively, compared with the original volume of the TOCN aerogels. This difference may be attributed to the more rigid sheet-like structure of $\mathrm{T} 2$, which was more stable during the pyrolysis process. T1 and T2 had similar density, while they exhibited different volume shrinkage ( 75 and $62 \% \mathrm{v} / \mathrm{v}$ for $\mathrm{T} 1$ and $\mathrm{T} 2$, respectively), leading to a density difference between $\mathrm{C} 1$ and $\mathrm{C} 2\left(8.8\right.$ and $\left.5.8 \mathrm{mg} \mathrm{cm}^{-3}\right)$ after pyrolysis. Compared with $\mathrm{T} 1$ and $\mathrm{T} 2$, the weight loss of both $\mathrm{C} 1$ and $\mathrm{C} 2$ after pyrolysis was about $78 \%$ due to the release of water, $\mathrm{CO}_{2}, \mathrm{CO}, \mathrm{CH}_{4}$ and some organics. ${ }^{28}$ The TOCN carbon aerogels showed ultralight properties (Fig. 2c), which are comparable to CNT sponges $\left(5-10 \mathrm{mg} \mathrm{cm}^{-3}\right)^{12}$ and graphene foam grown by CVD $\left(5 \mathrm{mg} \mathrm{cm}^{-3}\right) .{ }^{29}$ The porosity of $\mathrm{C} 1$ and $\mathrm{C} 2$ was calculated to be about $99.50 \%$ and $99.67 \%$, respectively, showing high porosity. From SEM images of carbon aerogels in Fig. $2 \mathrm{e}$ and $\mathrm{f}$, it is clear that the 3D porous structure and sheet-like structure was well maintained in $\mathrm{C} 1$ and $\mathrm{C} 2$.

\section{Surface area and porosity of carbon aerogels}

The $\mathrm{N}_{2}$ adsorption isotherms and the pore size distributions of the TOCN carbon aerogels are shown in Fig. 3a and b. With respect to the IUPAC classification, the isotherms for the samples of C1 and C2 are similar in shape to type II isotherms, indicating that an open surface is present in the TOCN carbon aerogels on which unrestricted monolayer-multilayer adsorption occurs. ${ }^{30}$ The sharp inflection points are present at a low relative pressure of about 0.1, showing the transition from micropore filling to multilayer adsorption. Then, the slow isotherm rise indicates multilayer adsorption of nitrogen on the exposed surface of the 3D structure in the carbon aerogels (Fig. 3a). ${ }^{31}$ This exposed surface makes the major contribution to oil absorption, rather than micropores or mesopores. Fig. $3 \mathrm{~b}$ shows the distribution of internal surface area versus pore sizes for the two carbon aerogel samples. Table 3 summarizes surface area parameters of $\mathrm{C} 1$ and $\mathrm{C} 2$ obtained from the BET tests. Both $\mathrm{C} 1$ and $\mathrm{C} 2$ have large mesopores being consistent with the $\mathrm{N}_{2}$ adsorption isotherms. The large specific surface of $\mathrm{C} 1$, which is larger than $\mathrm{C} 2$, should be attributed to the smaller ice crystals formed during the low temperature freezing process.

When the obtained TOCN carbon aerogels were immersed in water, air bubbles appeared around its surface (Fig. 2d), and after releasing the external force, TOCN carbon aerogels floated immediately without any absorption of water. This result indicated that the obtained TOCN carbon aerogels were hydrophobic. In addition, the contact angles of C1 and C2 were examined, where the water droplets deposited on the TOCN carbon aerogel surfaces were almost spherical with the contact angles of $139.6^{\circ}$ and $139.3^{\circ}$ (Table 3), respectively. Considering the 3D porous structure and higher surface area, $\mathrm{C} 1$ was chosen for following adsorption experiments.

\section{FTIR, XRD and XPS of aerogels}

The Fourier transform infrared (FTIR) spectra were used to investigate the chemical composition of TOCN and TOCN carbon aerogels. As shown in Fig. 4a, the FTIR spectrum of the

Table 2 Porosity and gasoline adsorption capacity of the TOCN carbon aerogels, after pyrolysis, from different TOCN concentrations

\begin{tabular}{|c|c|c|c|c|c|}
\hline TOCN concentration (wt\%) & & 0.5 & 1 & 1.5 & 2 \\
\hline \multirow[t]{2}{*}{ Porosity (\%) } & $\mathrm{C} 1$ & $99.75 \pm 0.01$ & $99.50 \pm 0.02$ & $99.26 \pm 0.02$ & $99.00 \pm 0.02$ \\
\hline & $\mathrm{C} 2$ & $99.83 \pm 0.01$ & $99.67 \pm 0.01$ & $99.50 \pm 0.02$ & $99.34 \pm 0.02$ \\
\hline
\end{tabular}



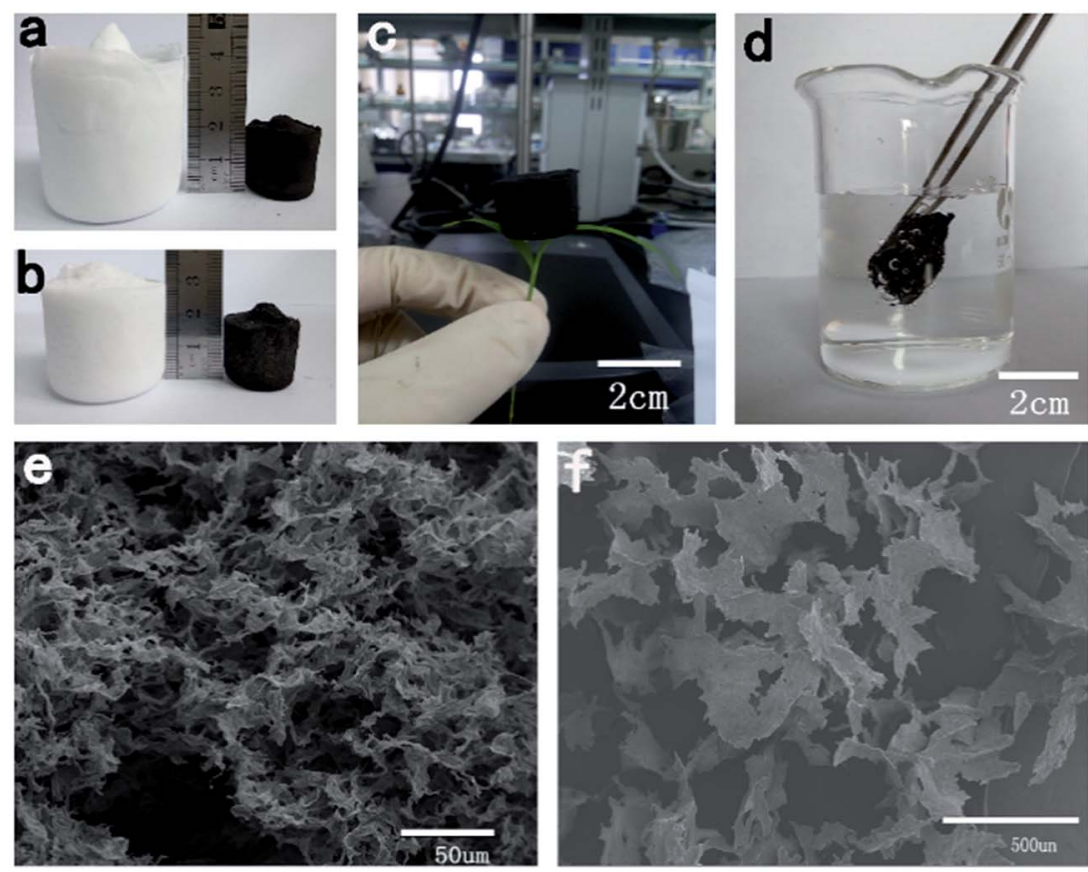

Fig. 2 (a) and (b) TOCN aerogels on the left $(T 1, T 2)$ and carbon aerogels on the right (C1, C2). (c) The TOCN carbon aerogels stand on the top of the grass blade in a stable manner. (d) A TOCN carbon aerogel sample immersed in water. (e) and (f) SEM images of carbon aerogels $\mathrm{C} 1$ and $\mathrm{C} 2$.
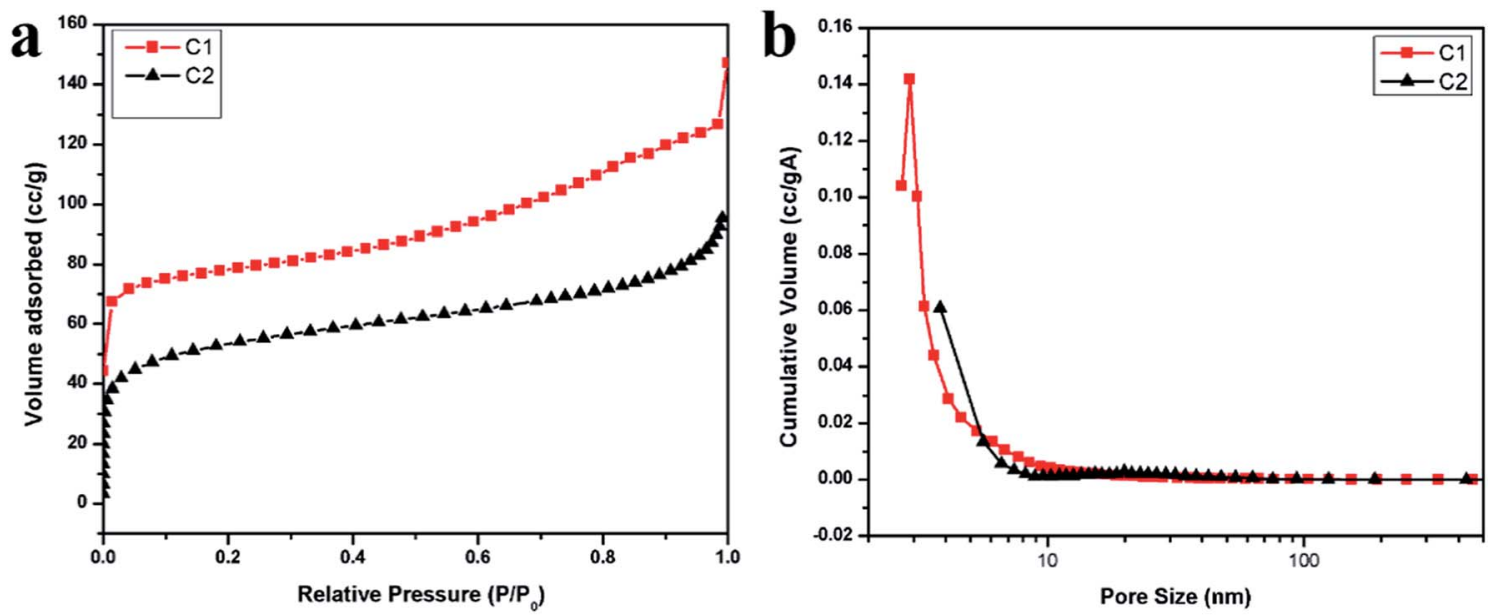

Fig. 3 (a) $\mathrm{N}_{2}$ adsorption isotherms and (b) the pore size distributions of $\mathrm{C} 1$ and $\mathrm{C} 2$.

original cellulose material and TOCN showed several peaks of hydrophilic functional groups, such as $\mathrm{C}=\mathrm{O}, \mathrm{C}-\mathrm{O}$, and $-\mathrm{OH}$. The peaks at $3340 \mathrm{~cm}^{-1}$ and $1047 \mathrm{~cm}^{-1}$ were attributed to the

Table 3 Density, porosity, and contact angle of the TOCN carbon aerogels

\begin{tabular}{lllll}
\hline Sample & $\begin{array}{l}\text { Density } \\
\left(\mathrm{mg} \mathrm{cm}^{-3}\right)\end{array}$ & Porous $(\%)$ & $\begin{array}{l}\text { BET surface } \\
\text { area }\left(\mathrm{m}^{2} \mathrm{~g}^{-1}\right)\end{array}$ & $\begin{array}{l}\text { Contact } \\
\text { angle }\left({ }^{\circ}\right)\end{array}$ \\
\hline $\mathrm{T} 1$ & $10 \pm 0.2$ & $99.33 \pm 0.02$ & - & - \\
$\mathrm{T} 2$ & $10 \pm 0.2$ & $99.33 \pm 0.02$ & - & - \\
$\mathrm{C} 1$ & $8.8 \pm 0.2$ & $99.50 \pm 0.02$ & 249.91 & $139.6 \pm 0.6$ \\
$\mathrm{C} 2$ & $5.8 \pm 0.1$ & $99.67 \pm 0.01$ & 161.16 & $139.3 \pm 0.5$
\end{tabular}

$\mathrm{O}-\mathrm{H}$ stretching vibration and the $\mathrm{C}-\mathrm{O}$ bond of cellulose, respectively,,$^{27}$ and the peaks at $2890 \mathrm{~cm}^{-1}$ and $1417 \mathrm{~cm}^{-1}$ corresponded to the $\mathrm{C}-\mathrm{H}$ stretching and bending of the $-\mathrm{CH}_{2}$ groups, respectively..$^{32}$ In addition, the peak at $1606 \mathrm{~cm}^{-1}$ was attributed to the $\mathrm{H}-\mathrm{O}-\mathrm{H}$ stretching vibration of absorbed water in the carbohydrate and the peak at $1730 \mathrm{~cm}^{-1}$ confirmed the presence of carboxylic acid groups. In contrast, peaks of hydrophilic functional groups, such as $\mathrm{C}=\mathrm{O}, \mathrm{C}-\mathrm{O}$, and $-\mathrm{OH}$, were all missing after pyrolysis, leading to the inference of hydrophobicity.

The X-ray diffraction (XRD) patterns (Fig. 4b) showed that both cellulose and TOCN aerogels exhibited the same diffraction peaks at $14.7,16.8$ and $22.7^{\circ}$, which corresponded to the (110), 

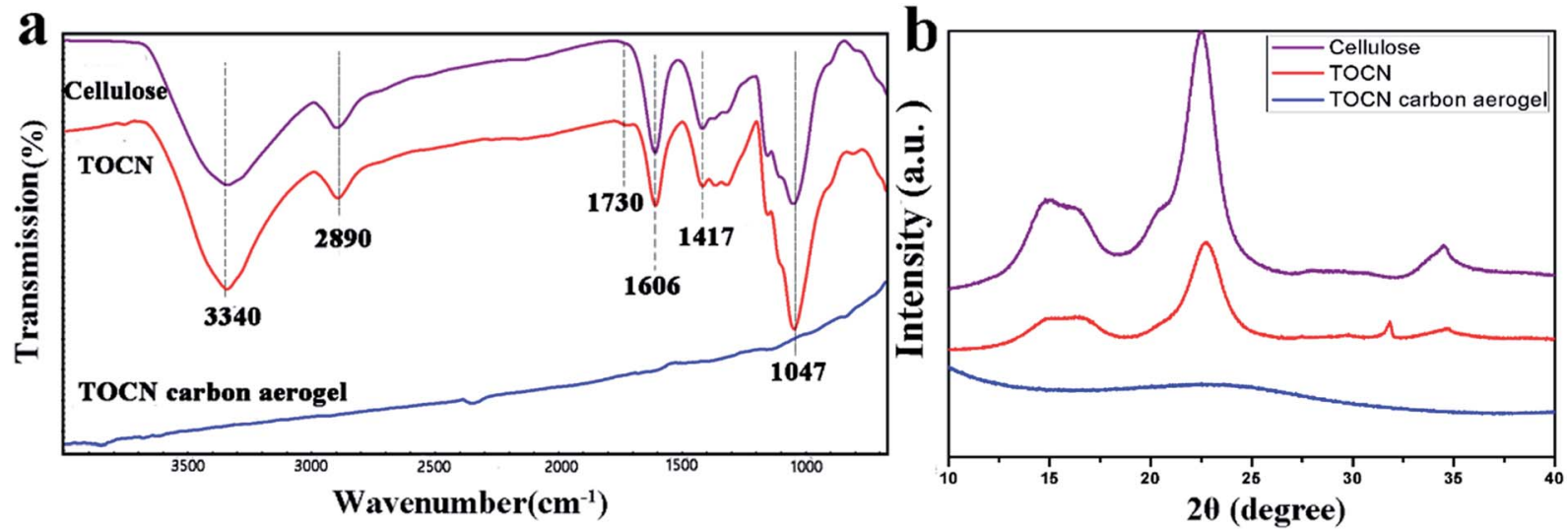

Fig. 4 (a) and (b) FTIR spectra and XRD patterns of the cellulose, the TOCN and the TOCN carbon aerogels.

(110) and (200) planes of cellulose, respectively. ${ }^{33}$ These planes are typical crystal patterns of cellulose I, indicating that the native crystal structure of cellulose was preserved during the TEMPO oxidation process. After pyrolysis at $1000{ }^{\circ} \mathrm{C}$, the peaks of TOCN aerogels almost diminish, suggesting that the crystalline structure of native cellulose was disrupted. TOCN carbon aerogels show a broad peak at $\sim 23^{\circ}$, indicating the formation of an amorphous phase. ${ }^{34}$ The elemental composition and bonding configurations of the TOCN carbon aerogels were investigated by XPS. As shown in Fig. 5, the $\mathrm{C} 1 \mathrm{~s}$ and $\mathrm{O} 1 \mathrm{~s}$ peaks are observed at 284.3 and $531.4 \mathrm{eV}$, respectively. The atom ratios of $\mathrm{C}$ to $\mathrm{O}$ are 1.39 and 27.57 for TOCN and the TOCN carbon aerogels, respectively, which are due to dehydration, decarboxylation and decarbonylation reaction during the pyrolysis process. ${ }^{35}$

\section{Organic solvents absorption performance of TOCN carbon aerogels}

Owing to its surface hydrophobicity and high porosity, the TOCN carbon aerogel was an ideal candidate for highly efficient

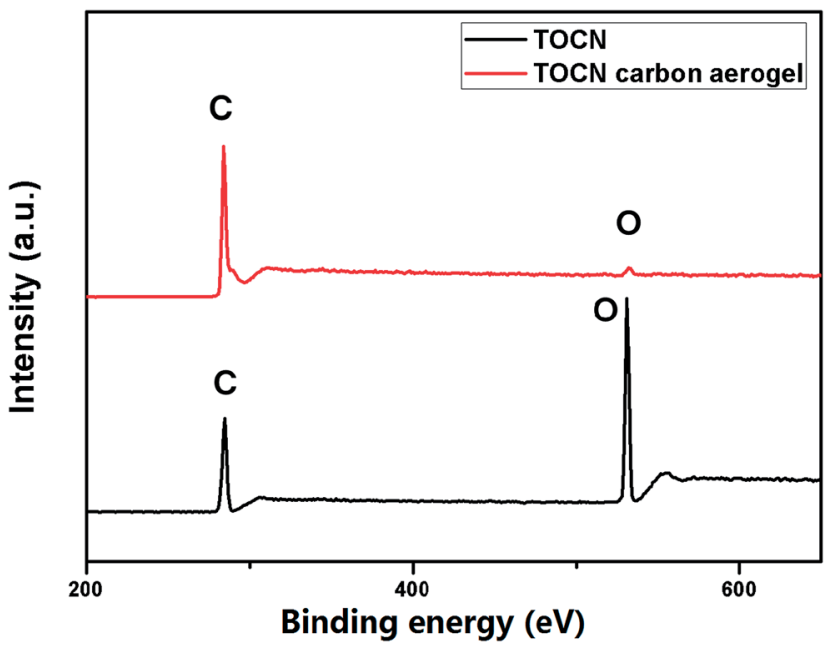

Fig. 5 X-ray photoelectron spectroscopy (XPS) analysis of TOCN and the TOCN carbon aerogels. separation of organic solvents. As shown in Fig. 6a and Video $\mathrm{S} 1, \dagger$ when a hydrophobic TOCN carbon aerogel was immersed into the $n$-hexane and water mixture $(1: 20 \mathrm{v} / \mathrm{v}$, colored with Sudan III), it floated on the water surface and selectively
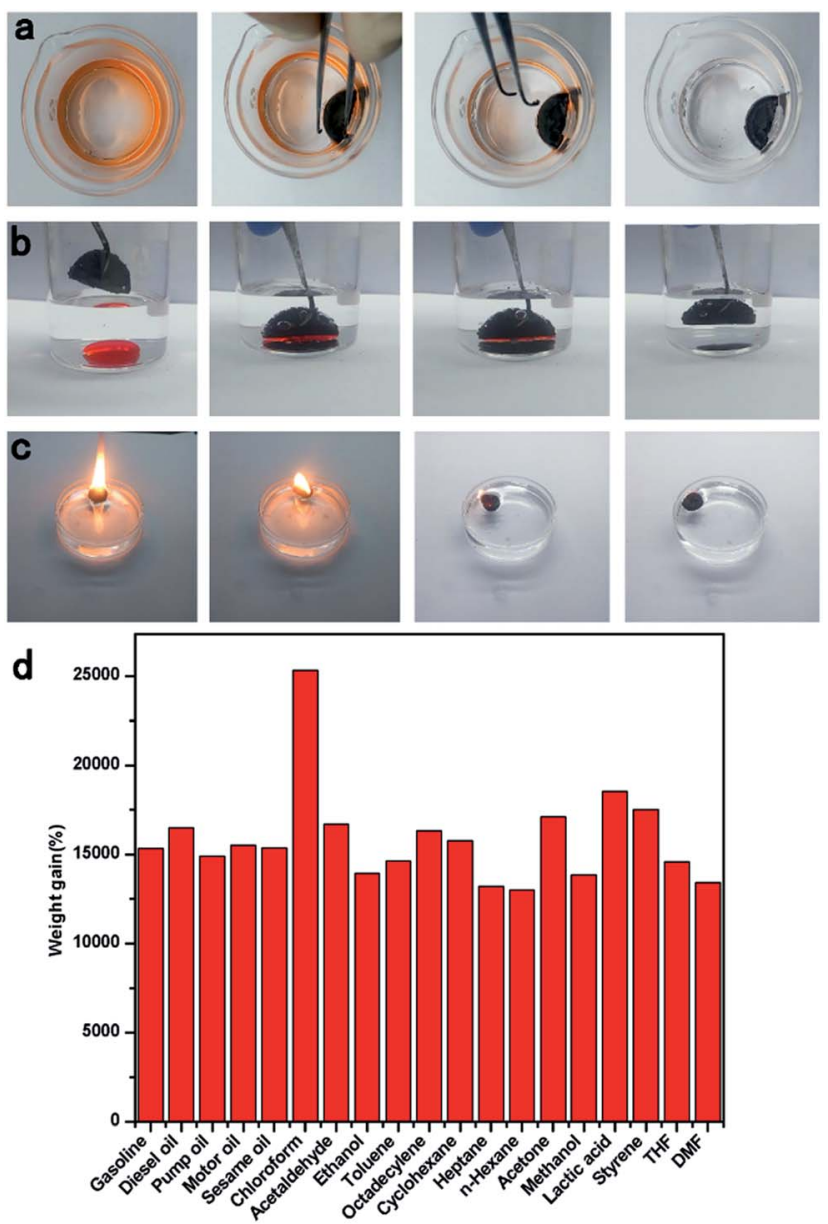

Fig. 6 (a) A layer of $n$-hexane was absorbed completely by a TOCN carbon aerogel sample in 10 seconds. (b) The sorption process of chloroform. (c) The carbon aerogels absorbed with gasoline burning off and floating on the water. (d) Absorption capacity of the TOCN carbon aerogels for various organic solvents. 
absorbed $n$-hexane completely in 10 seconds, exhibiting excellent selectivity and adsorption rate. In addition, other high-density organic solvents like chloroform (stained with Sudan III), lying at the bottom of the water can also be selectively absorbed by the TOCN carbon aerogels in 6 seconds (Fig. 6b and Video S2 $\dagger$ ).

As shown in Fig. 6d, the sorption capacity of TOCN carbon aerogels with a variety of organic solvents has been investigated, including gasoline, toluene, ethanol, and acetone. The weight of absorbed substance per unit weight of the dried TOCN carbon aerogels was calculated to evaluate the sorption capacity quantitatively. As shown in Table 4, the sorption capacity of TOCN carbon aerogels ranged from 110 to 260 times its own weight depending on the density of the organic solvent. It is clear that the TOCN carbon aerogels show much higher

Table 4 Comparison of various absorption materials

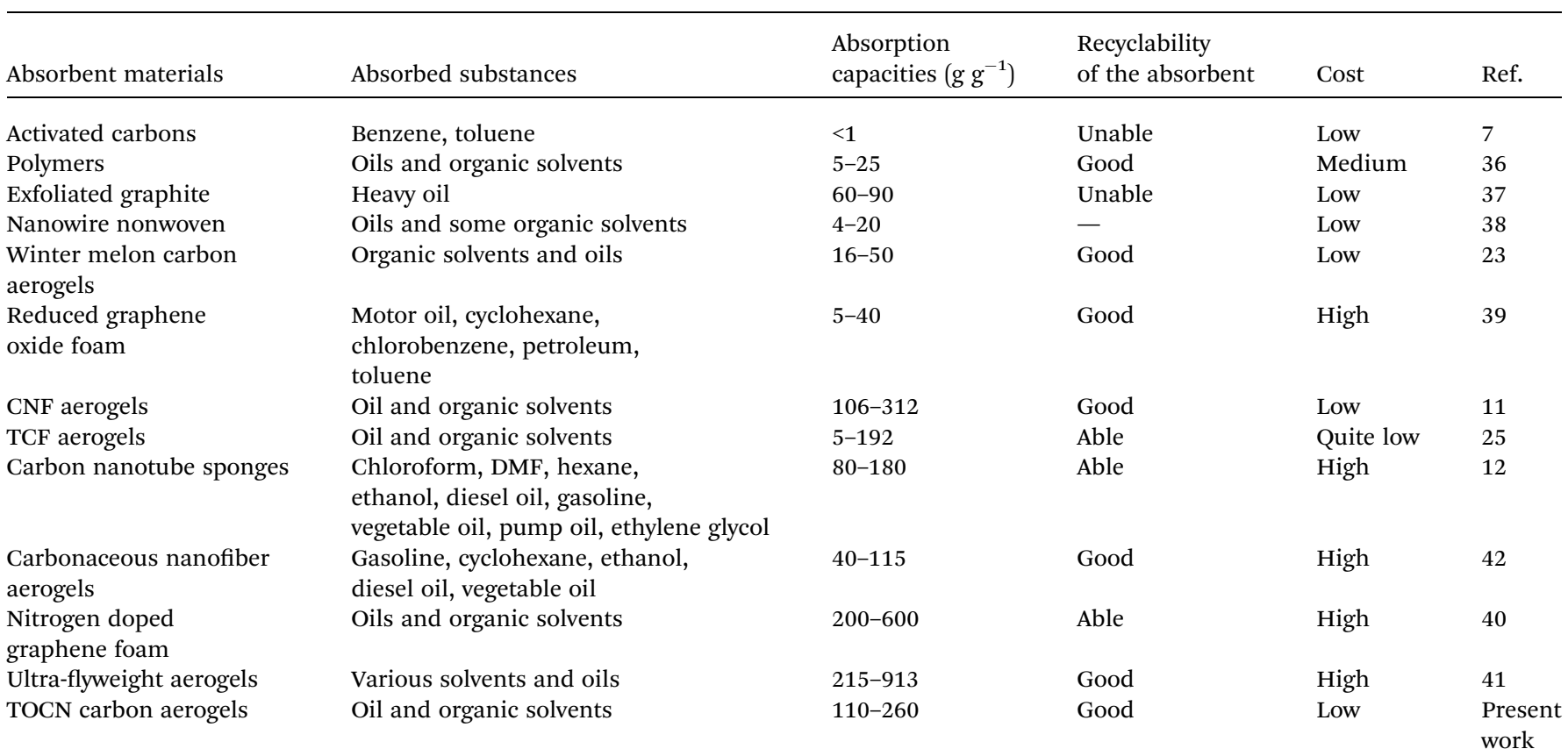

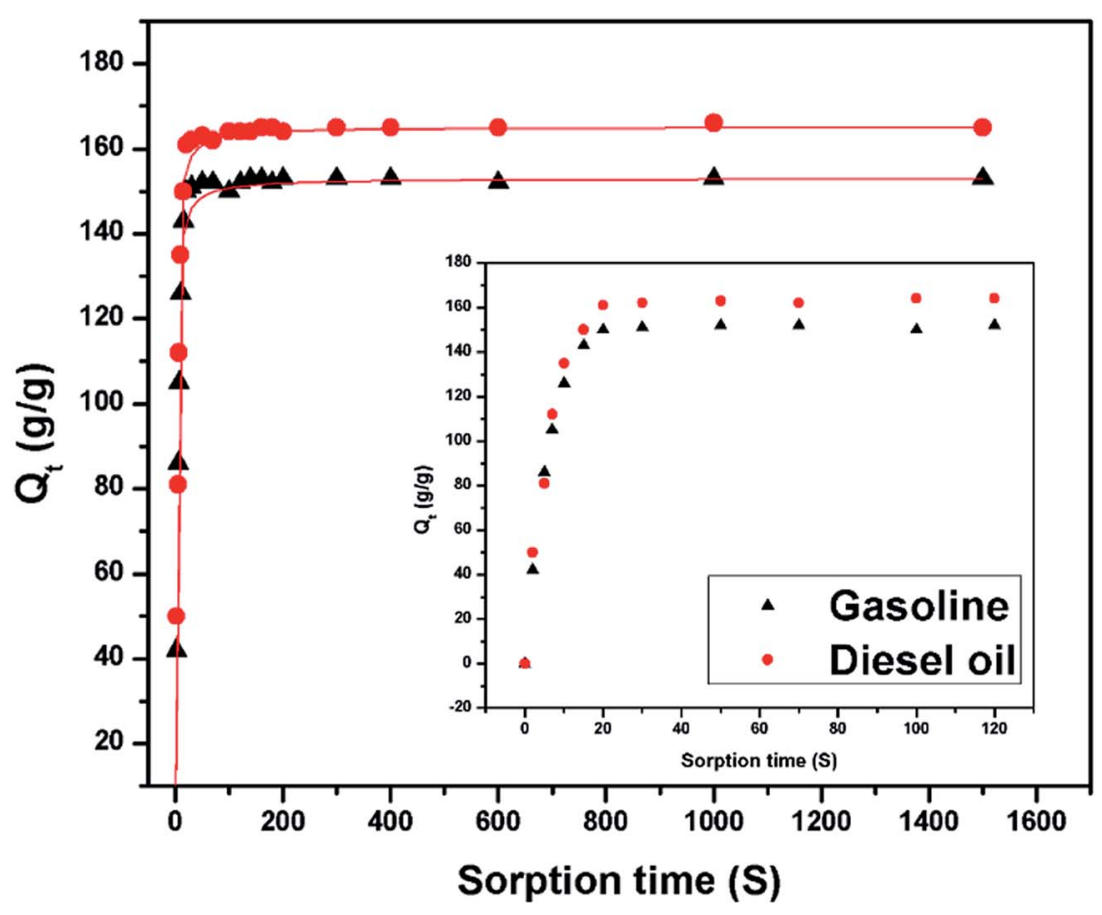

Fig. 7 Sorption kinetics of gasoline and diesel oil absorbed into the TOCN carbon aerogel. 
sorption capacity than several previously reported absorbents, such as activated carbon ( $<1$ times), ${ }^{7}$ polymers (5-25 times), ${ }^{36}$ exfoliated graphite (60-90 times), ${ }^{37}$ nanowire membrane (4-20 times), ${ }^{38}$ winter melon carbon aerogels (16-50 times), ${ }^{23}$ and reduced graphene oxide foam (5-40 times). ${ }^{39}$ Although the sorption capacity of TOCN carbon aerogels was still lower than that of nitrogen-doped graphene foam (200-600 times) ${ }^{40}$ and ultra-flyweight aerogels (215-743 times), ${ }^{\mathbf{4 1}}$ the fabrication method of TOCN carbon aerogels was much simpler and its precursor material was cheaper and nontoxic, indicating that TOCN carbon aerogels could be a cost-effective and promising absorbent for removal of oil and various organic solvents.

\section{Oil absorption kinetics}

Fig. 7 shows the sorption capacity $\left(Q_{t}\right)$ of gasoline and diesel oil in TOCN carbon aerogels plotted as a function of absorption time. At the beginning of absorption, the weight of the TOCN carbon aerogels rapidly increased, indicating the capillary phenomenon. The oil quickly permeated into the pores of carbon aerogels by capillary action. ${ }^{31}$ The maximum absorbency of diesel oil was higher than that of gasoline because the density of diesel oil $\left(0.843 \mathrm{~g} \mathrm{~cm}^{-3}\right)$ was higher than that of gasoline $\left(0.722 \mathrm{~g} \mathrm{~cm}^{-3}\right)$. The time for the TOCN carbon aerogels to reach the saturated sorption capacity was about $20 \mathrm{~s}$, indicating that the TOCN carbon aerogel was a candidate material for oil absorption with a fast absorption rate.

Pseudo-first order and pseudo-second order models are commonly used for oil absorption studies. ${ }^{\mathbf{4 3 , 4 4}}$ The sorption

Table 5 The gasoline and diesel oil's properties and fitting parameters of the sorption kinetics

\begin{tabular}{llllll}
\hline Oil & $\begin{array}{l}\text { Density } \\
\left(\mathrm{g} \mathrm{cm}^{-3}\right)\end{array}$ & $\begin{array}{l}\text { Viscosity } \\
(\mathrm{cST})\end{array}$ & $K_{1}$ & $K_{2}$ & $Q_{\mathrm{s}}\left(\mathrm{g} \mathrm{g}^{-1}\right)$ \\
\hline Gasoline & 0.722 & 0.76 & 0.1949 & 0.1806 & $153 \pm 3$ \\
Diesel oil & 0.843 & 4.7 & 0.0048 & 0.0045 & $165 \pm 3$
\end{tabular}
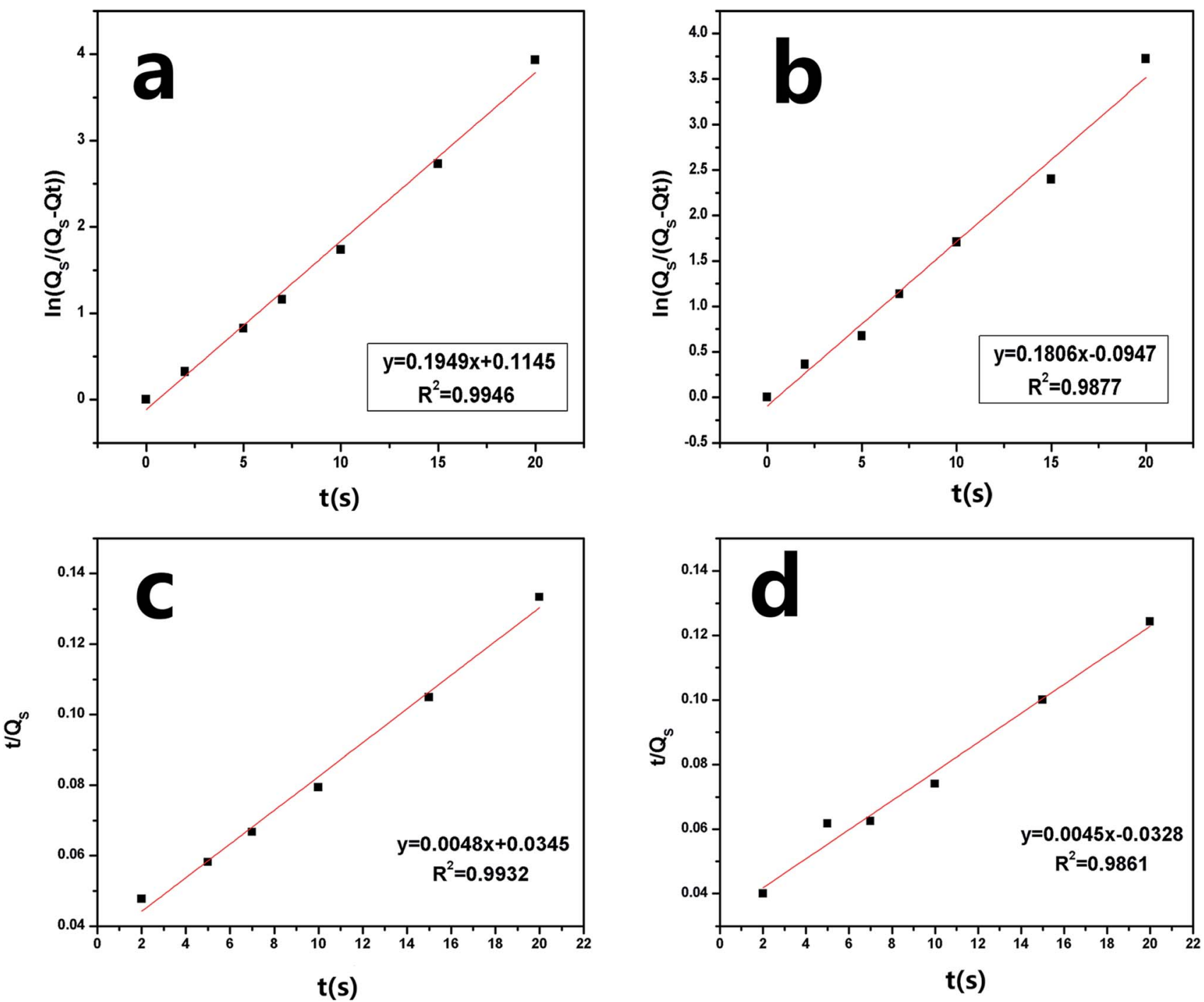

Fig. 8 Pseudo-first order absorption linear fitting of (a) gasoline and (b) diesel oil and pseudo-second order absorption linear fitting of (c) gasoline (d) diesel oil of the TOCN carbon aerogel. 
kinetics of the TOCN carbon aerogels were analyzed based on both these models. After integration, the pseudo-first order equation can be obtained: ${ }^{31}$

$$
\ln \frac{Q_{\mathrm{s}}}{Q_{\mathrm{s}}-Q_{t}}=k_{1} t
$$

Furthermore, the pseudo-second order equation can be converted into a linear form: ${ }^{45}$

$$
\frac{t}{Q_{t}}=\frac{1}{Q_{\mathrm{s}}} t+\frac{1}{k_{2} Q_{\mathrm{s}}^{2}}
$$

where $Q_{\mathrm{s}}\left(\mathrm{g} \mathrm{g}^{-1}\right)$ is the saturated oil absorption, $Q_{t}$ is the oil absorbed at time $t, k_{1}$ is the absorption rate constant determined from the slope of $\ln \left[Q_{\mathrm{s}} /\left(Q_{\mathrm{s}}-Q_{t}\right)\right]$ versus $t$ plot and $k_{2}$ is the kinetic rate constant determined from plotting $\left(t / Q_{t}\right)$ versus $t$.

Fig. 8a and b shows the plots of $\ln \left[Q_{\mathrm{s}} /\left(\mathrm{Q}_{\mathrm{s}}-\mathrm{Q}_{t}\right)\right]$ versus time $t$ using the pseudo-first order model, while Fig. 8c and d displays the plots of $\left(t / Q_{t}\right)$ against $t$ using the pseudo-second order model. In the inset of Fig. 8, the sorption rate constants $k_{1}$ and $k_{2}$ and the correlation coefficient $R^{2}$ are shown. The results show that the absorption rate constants $k_{1}$ and $k_{2}$ for the gasoline are greater than those for diesel oil (Table 5), implying that the oil absorption of gasoline is faster than diesel oil due to its lower viscosity. Based on the pseudo-second order equation, the fitting results are plotted with a red line in Fig. 7, which shows excellent agreement with the experimental data.

\section{Absorption recyclability of TOCN carbon aerogels}

Since most pollutants are either useful or expensive raw materials, such as crude oil and toluene, the recyclability of absorbents and the recoverability of pollutants are key criteria for oil and chemical cleanup. TOCN carbon aerogels exhibit excellent fire-resistance when exposed to the flame of an alcohol burner, resulting in no burning of the aerogel (the temperature of the flame of the alcohol lamp is about 400-500 $\left.{ }^{\circ} \mathrm{C}\right)$. This similar phenomenon was reported in previous literature, ${ }^{\mathbf{1 1 , 3 1 , 4 6}}$ which may have been due to the high porosity of aerogels allowing for the quick removal of heat during combustion. ${ }^{47}$ Considering the fire-resistance and the high oil absorption capacity of the TOCN carbon aerogels, we used direct combustion to remove the gasoline and alcohol by an absorption/combustion method. In addition, cyclic absorption-combustion cycles were also performed. For gasoline and ethanol (Fig. 9b and c), 64\% and $87 \%$ of the original TOCN carbon aerogel absorption capacity could be retained after 5 cycles, respectively. This result may be ascribed to the collapse of the porous structure and the shrinkage of the TOCN carbon

\section{a}
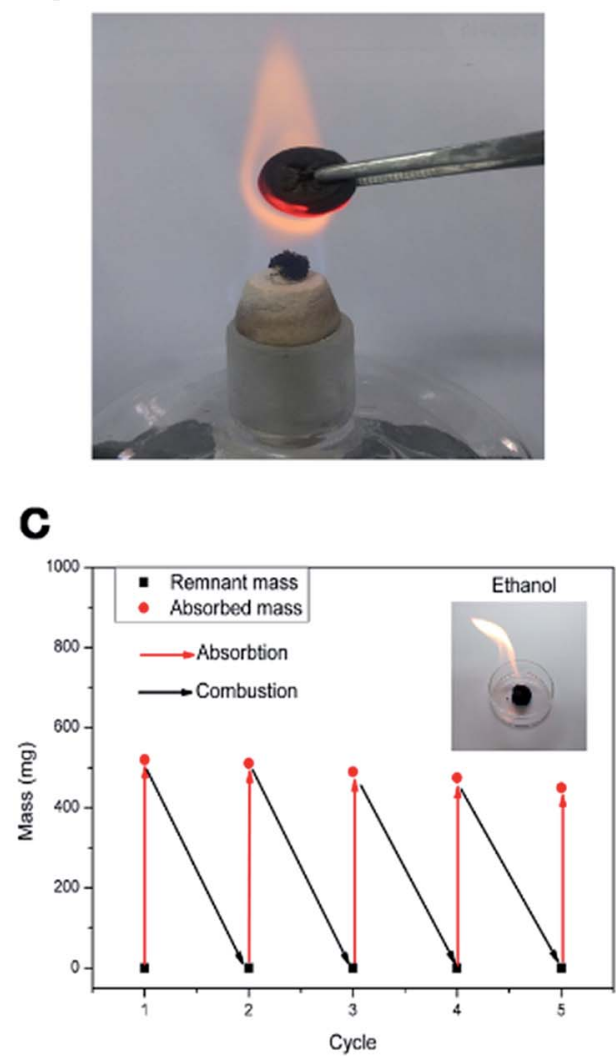

b

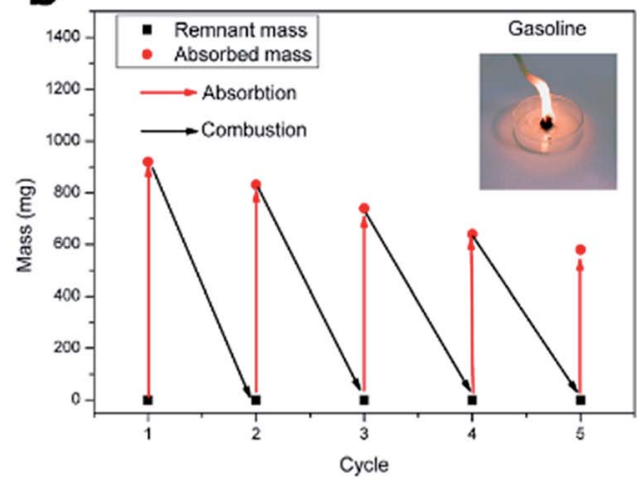

d

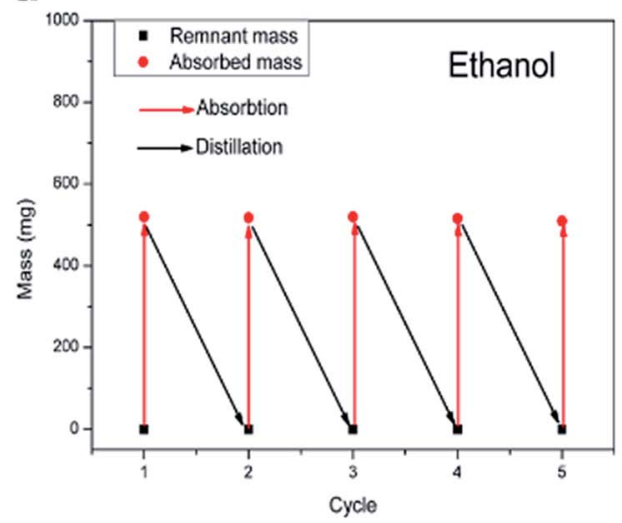

Fig. 9 (a) Image of TOCN carbon aerogels glowing in a flame. (b) Recyclability of TOCN carbon aerogels for absorption of gasoline. (c) Recyclability of TOCN carbon aerogels for absorption of ethanol when using the direct combustion method. (d) Recyclability of TOCN carbon aerogels for absorption of ethanol when using the distillation method. 

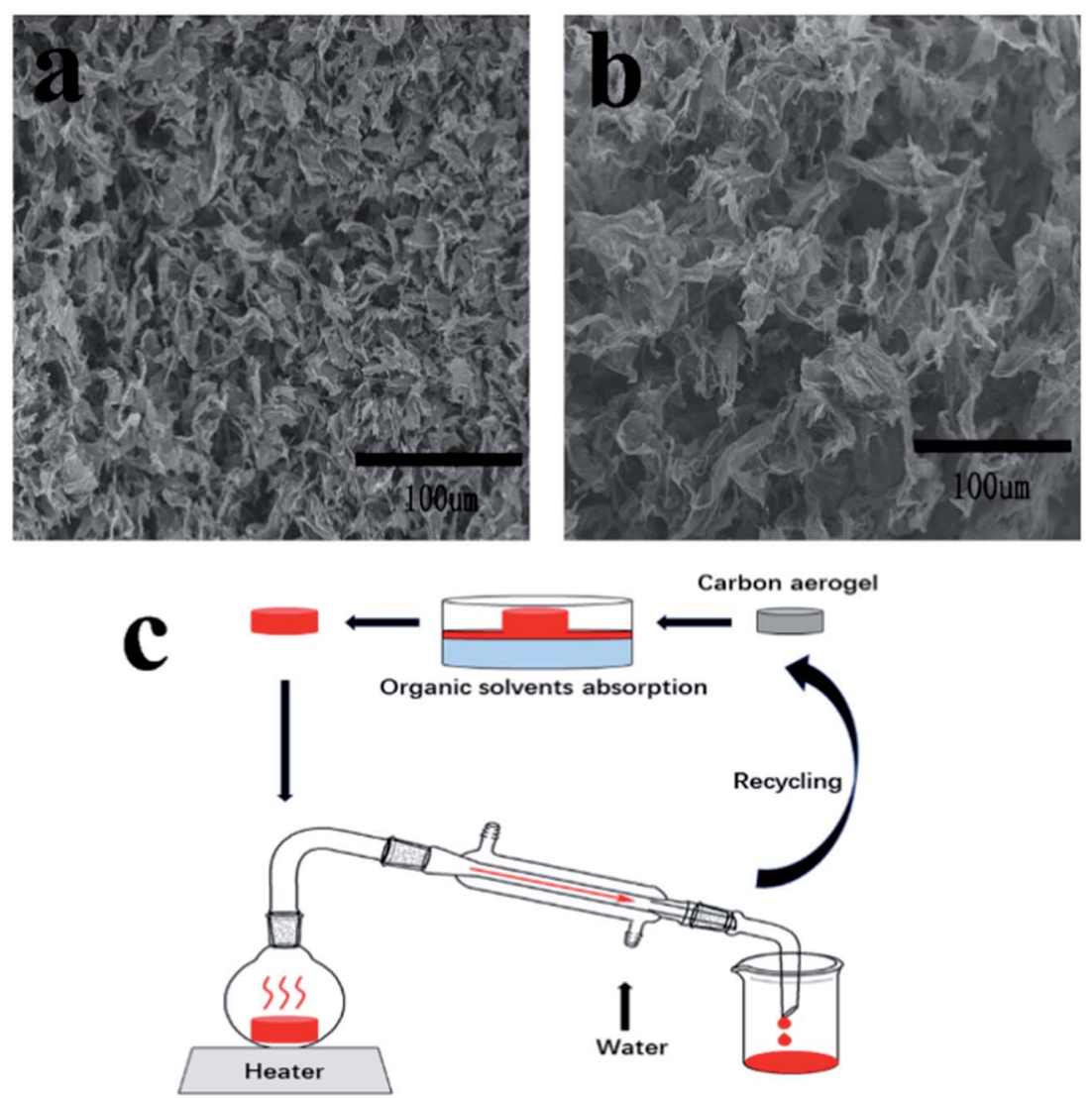

Fig. 10 (a) and (b) SEM images of TOCN carbon aerogels after five absorption-combustion cycles and absorption-distillation cycles. (c) Schematic diagram of TOCN carbon aerogels recycling process.

aerogels after the combustion process (Fig. 9a) compared with the original carbon aerogels (Fig. 2e). Generally, the thin oil layer floating on the water surface cannot be combusted directly due to being difficult to gasify. However, TOCN carbon aerogels float on the water and can absorb the oil quickly and act as a porous carbon wick that efficiently facilitates the combustion. After the combustion finishes and the flame extinguishes, TOCN carbon aerogels still float on the water surface (Fig. 6c). Therefore, TOCN carbon aerogels may have potential applications in dealing with oil spill accidents.

It should be noted that combustion is a simple but wasteful method; the collection and recycling of these organic solvents would be more economical and environmentally-friendly. As illustrated in Fig. 10c, a distillation procedure is employed to recover TOCN carbon aerogels and recycle the low boiling point pollutants, unlike the combustion process that would waste the precious or toxic organic solvents. After absorption, the TOCN carbon aerogels were heated to the boiling point $\left(80^{\circ} \mathrm{C}\right)$ to release the absorbed alcohol. Then, we collected the vapor of the alcohol for recycling. The result indicated that no significant decrease in absorption capacity of TOCN carbon aerogels was found after five absorption-distillation cycles, which may be ascribed to the relatively gentle treatment process. The pore size and structures were retained after the distillation process (Fig. 10b) compared with the original carbon aerogels (Fig. 2e). Thus, all these benefits suggest that the absorption-distillation is a more valuable approach to recover the solvent in comparison with the absorptioncombustion cycle.

\section{Conclusions}

In summary, we have successfully prepared carbon-based aerogels from TEMPO-oxidized cellulose nanofibers (TOCN) by pyrolyzing TOCN aerogels at $1000{ }^{\circ} \mathrm{C}$ under nitrogen flow. The TOCN carbon aerogels show excellent porosity (99.5\%) and hydrophobic properties with a contact angle of $139.6^{\circ}$. This super-absorbent was ultralight with a density of $8.8 \mathrm{mg} \mathrm{cm}^{-3}$ and exhibited excellent oil/water selectivity and high absorption capacity varying from 110 to $260 \mathrm{~g} \mathrm{~g}^{-1}$ depending on the density of the organic solvents. In addition, the excellent fire-resistance allowed the carbon aerogels to be reusable after direct combustion of the absorbed solvent. These unique properties of TOCN carbon aerogels, along with the advantages of using a natural, low-cost and sustainable material, enable TOCN carbon aerogels to have potential applications in environmental pollutant treatment in the future.

\section{Conflict of interest}

The authors declare no competing financial interest. 


\section{Acknowledgements}

This work was financially supported by Fundamental Research Funds for the Central Universities (2017ZY35), Chinese Ministry of Education (113014A), National Natural Science Foundation of China (21404011, 21674013) and State Key Laboratory of Pulp and Paper Engineering (201750).

\section{References}

1 H. M. Choi and R. M. Cloud, Environ. Sci. Technol., 1992, 26, 772-776.

2 M. A. Shannon, P. W. Bohn, M. Elimelech, J. G. Georgiadis, B. J. Marinas and A. M. Mayes, Nature, 2008, 452, 301-310.

3 M. O. Adebajo, R. L. Frost, J. T. Kloprogge, O. Carmody and S. Kokot, J. Porous Mater., 2003, 10, 159-170.

4 D. Bastani, A. A. Safekordi, A. Alihosseini and V. Taghikhani, Sep. Purif. Technol., 2006, 52, 295-300.

5 T. Sakthivel, D. L. Reid, I. Goldstein, L. Hench and S. Seal, Environ. Sci. Technol., 2013, 47, 5843-5850.

6 M. Radetic, V. Ilic, D. Radojevic, R. Miladinovic, D. Jocic and P. Jovancic, Chemosphere, 2008, 70, 525-530.

7 M. Lillo-Ródenas, D. Cazorla-Amorós and A. Linares-Solano, Carbon, 2005, 43, 1758-1767.

8 S. S. Banerjee, M. V. Joshi and R. V. Jayaram, Chemosphere, 2006, 64, 1026-1031.

9 A. C. Pierre and G. M. Pajonk, Chem. Rev., 2002, 102, 42434266.

10 A. M. Elkhatat and S. A. Al-Muhtaseb, Adv. Mater., 2011, 23, 2887-2903.

11 Z. Y. Wu, C. Li, H. W. Liang, J. F. Chen and S. H. Yu, Angew. Chem., 2013, 52, 2925-2929.

12 X. Gui, J. Wei, K. Wang, A. Cao, H. Zhu, Y. Jia, Q. Shu and D. Wu, Adv. Mater., 2010, 22, 617-621.

13 F. Yavari, Z. P. Chen, A. V. Thomas, W. C. Ren, H. M. Cheng and N. Koratkar, Sci. Rep., 2011, 1, 166.

14 X.-C. Dong, H. Xu, X.-W. Wang, Y.-X. Huang, M. B. ChanPark, H. Zhang, L.-H. Wang, W. Huang and P. Chen, ACS Nano, 2012, 6, 3206-3213.

15 Z.-S. Wu, S. Yang, Y. Sun, K. Parvez, X. Feng and K. Müllen, J. Am. Chem. Soc., 2012, 134, 9082-9085.

16 H. W. Liang, Q. F. Guan, L. F. Chen, Z. Zhu, W. J. Zhang and S. H. Yu, Angew. Chem., 2012, 51, 5101-5105.

17 R. Pekala, J. Mater. Sci., 1989, 24, 3221-3227.

18 X. Dong, J. Chen, Y. Ma, J. Wang, M. B. Chan-Park, X. Liu, L. Wang, W. Huang and P. Chen, Chem. Commun., 2012, 48, 10660-10662.

19 R. J. Moon, A. Martini, J. Nairn, J. Simonsen and J. Youngblood, Chem. Soc. Rev., 2011, 40, 3941-3994.

20 M. A. S. Azizi Samir, F. Alloin and A. Dufresne, Biomacromolecules, 2005, 6, 612-626.

21 H. Koga, T. Kitaoka and A. Isogai, Molecules, 2015, 20, 14951508.
22 D. Klemm, F. Kramer, S. Moritz, T. Lindstrom, M. Ankerfors, D. Gray and A. Dorris, Angew. Chem., 2011, 50, 5438-5466.

23 Y. Q. Li, Y. A. Samad, K. Polychronopoulou, S. M. Alhassan and K. Liao, ACS Sustainable Chem. Eng., 2014, 2, 1492-1497.

24 R. L. Liu, Y. Liu, X. Y. Zhou, Z. Q. Zhang, J. Zhang and F. Q. Dang, Bioresour. Technol., 2014, 154, 138-147.

25 H. Bi, Z. Yin, X. Cao, X. Xie, C. Tan, X. Huang, B. Chen, F. Chen, Q. Yang, X. Bu, X. Lu, L. Sun and H. Zhang, Adv. Mater., 2013, 25, 5916-5921.

26 Y. Okita, T. Saito and A. Isogai, Biomacromolecules, 2010, 11, 1696-1700.

27 W. Chen, Q. Li, Y. Wang, X. Yi, J. Zeng, H. Yu, Y. Liu and J. Li, ChemSusChem, 2014, 7, 154-161.

28 H. Yang, R. Yan, H. Chen, D. H. Lee and C. Zheng, Fuel, 2007, 86, 1781-1788.

29 Z. Chen, W. Ren, L. Gao, B. Liu, S. Pei and H.-M. Cheng, Nat. Mater., 2011, 10, 424-428.

30 Z. Ryu, J. Zheng, M. Wang and B. Zhang, Carbon, 1999, 37, 1257-1264.

31 Y. Meng, T. M. Young, P. Liu, C. I. Contescu, B. Huang and S. Wang, Cellulose, 2014, 22, 435-447.

32 C. Zhang, R. Z. Zhang, Y. Q. Ma, W. B. Guan, X. L. Wu, X. Liu, H. Li, Y. L. Du and C. P. Pan, ACS Sustainable Chem. Eng., 2015, 3, 396-405.

33 S. Y. Oh, D. I. Yoo, Y. Shin, H. C. Kim, H. Y. Kim, Y. S. Chung, W. H. Park and J. H. Youk, Carbohydr. Res., 2005, 340, 23762391.

34 M. Noh, Y. Kwon, H. Lee, J. Cho, Y. Kim and M. G. Kim, Chem. Mater., 2005, 17, 1926-1929.

35 C. Șerbănescu, Chem. Pap., 2014, 68, 847-860.

36 A. Li, H. X. Sun, D. Z. Tan, W. J. Fan, S. H. Wen, X. J. Qing, G. X. Li, S. Y. Li and W. Q. Deng, Energy Environ. Sci., 2011, 4, 2062-2065.

37 M. Toyoda and M. Inagaki, Carbon, 2000, 38, 199-210.

38 J. Yuan, X. Liu, O. Akbulut, J. Hu, S. L. Suib, J. Kong and F. Stellacci, Nat. Nanotechnol., 2008, 3, 332-336.

39 Z. Niu, J. Chen, H. H. Hng, J. Ma and X. Chen, Adv. Mater., 2012, 24, 4144-4150.

40 Y. Zhao, C. Hu, Y. Hu, H. Cheng, G. Shi and L. Qu, Angew. Chem., 2012, 124, 11533-11537.

41 H. Sun, Z. Xu and C. Gao, Adv. Mater., 2013, 25, 2554-2560. 42 H. W. Liang, Q. F. Guan, L. F. Chen, Z. Zhu, W. J. Zhang and S. H. Yu, Angew. Chem., Int. Ed., 2012, 51, 5101-5105.

43 N. E. Thompson, G. Emmanue, K. J. Adagadzu and N. B. Yusuf, Arch. Appl. Sci. Res., 2010, 2, 142-151.

44 B. Wu and M. Zhou, Waste Manage., 2009, 29, 355-359.

45 Y. Chen and D. Zhang, Chem. Eng. J., 2014, 254, 579-585.

46 Z. Y. Wu, C. Li, H. W. Liang, Y. N. Zhang, X. Wang, J. F. Chen and S. H. Yu, Sci. Rep., 2014, 4, 4079.

47 J. Li, J. Li, H. Meng, S. Xie, B. Zhang, L. Li, H. Ma, J. Zhang and M. Yu, J. Mater. Chem. A, 2014, 2, 2934. 